\title{
Linx
}

Revue des linguistes de l'université Paris X Nanterre

$59 \mid 2008$

Les conjonctions en diachronie : parcours sémantiques

\section{La naissance des conjonctions de subordination en hongrois}

On the emergence of adverbial subordinators in Hungarian

\section{Anna Sőrés}

\section{CpenEdition}

\section{Journals}

Édition électronique

URL : http://journals.openedition.org/linx/631

DOI : 10.4000/linx.631

ISSN : 2118-9692

Éditeur

Presses universitaires de Paris Nanterre

Édition imprimée

Date de publication : 1 décembre 2008

Pagination : 133-148

ISSN : 0246-8743

Référence électronique

Anna Sörés, "La naissance des conjonctions de subordination en hongrois », Linx [En ligne], 59 | 2008, mis en ligne le 01 janvier 2012, consulté le 19 avril 2019. URL : http://journals.openedition.org/linx/631 ; DOI : 10.4000/linx.631 


\title{
La naissance des conjonctions de subordination en hongrois
}

\author{
Anna Sörés \\ Université Lyon 2- Laboratoire ICAR
}

Cet article se propose de 1) présenter le processus de la naissance de la subordination conjonctive dans une langue où de telles constructions étaient absentes à l'origine et 2) trouver la place du hongrois dans la typologie de Kortmann (1997). La description du processus pourrait contribuer au travail effectué sur le français. L'observation du hongrois, complétée par des données de travaux translinguistiques sur le sujet, permettra de chercher et de trouver des parallélismes. En effet, il a été observé (Kortmann 1997, 2001) que les langues européennes, dans le sens géographique du terme, ou plus précisément dans l'ensemble appelé Européen Moyen Type (Standard Average European), et surtout celles qui ont élaboré une langue littéraire sont particulièrement riches en conjonctions de subordination. C'est donc le point qui permet de joindre les observations sur le hongrois qui n'est pas en relation génétique avec le français mais qui partage certains traits que nous pourrions appeler « européismes ».

Avant de commencer l'analyse concrète, il paraît utile de chercher une définition générale qui serait utilisable dans notre cas où il s'agit d'une langue de structure différente de celle du français. Nous allons considérer les conjonctions de subordination (ou : adverbiales) comme des relateurs (Hagège 2001) ayant pour fonction de relier une partie de la phrase à une autre en mettant l'une dans la dépendance de l'autre. Dans cette fonction de relateur, les conjonctions sont comparables aux prépositions qui introduisent des groupes prépositionnels (Creissels 2006, II : 193) et qui participent à la construction du verbe en qualité d'obliques.

Notons pour terminer l'introduction que dans l'analyse typologique à laquelle nous allons nous référer (Kortmann 1997, 2001), l'accent est mis sur l'importance des conjonctions dans le langage écrit. Toutefois, certains s'interrogent (Bat-Zeev et Kemmer 1988) sur leur fonction dans le discours. En effet (selon Creissels 2006, II : 184) on peut se demander à partir de quel moment on peut considérer que les contraintes auxquelles obéissent les enchaînements d'unités phrastiques cessent d'être la pure et simple conséquence d'un principe discursif de cohérence pour devenir des règles de syntaxe. La présente approche se concentrera sur la langue écrite. 


\section{Rapports génétiques, périodisation, documentation}

Le hongrois est une langue âgée d'environ 3000 ans. Il fait partie de la branche finno-ougrienne des langues ouraliennes. En ce qui concerne la périodisation dont la connaissance sera important pour la description, nous adoptons celle de Pusztai et Kiss $(2000,16-17)$ car sa terminologie peut être facilement mise en correspondance avec les habitudes de périodisation d'autres langues, ce qui n'est pas toujours le cas dans les ouvrages plus traditionnels. On distingue les époques suivantes :

- Proto-hongrois : de 1000 av. JC. environ à la fin du 9e (896, arrivée des Hongrois dans le bassin des Carpates);

- Ancien hongrois : de la fin du 9e jusqu'au milieu du 16e (1526, défaite face aux Turcs);

- Moyen hongrois : du milieu du $16^{e}$ à la fin du XVIII e (1772, début de l'époque des Lumières);

- Hongrois moderne: de la fin du 18e jusqu'à nos jours, avec une césure possible à partir de 1920, à cause de la scission de la population (suite aux traités de Trianon après la guerre 14-18).

Les documents que nous considérons comme les premières sources écrites sont des copies, (appelés « codex») les originaux datent probablement de la fin du $12^{\mathrm{e}}$, début du $13^{\mathrm{e}}$ siècle. Les plus connus, cités dans les grammaires historiques du hongrois sont les suivants : Halotti Beszéd (Oraison funèbre) vers 1192-1195, Ómagyar Mária siralom (Planctus Sanctae Mariae) vers 1300, Gyulafehérvári sorok (lignes de Gyulafehérvár) 1310-1320, Königsbergi töredék (Fragments de Königsberg) vers $1350^{1}$.

Notons encore quelques propriétés du hongrois : il s'agit d'une langue majoritairement agglutinante, il a l'ordre SVO dans la phrase mais il est de type OV concernant les autres structures.

\section{Présentation chronologique de l'apparition des conjonctions de subordination}

\subsection{Généralités}

En ce qui concerne les subordonnées en général, on accepte (Bereczki 1996, 94) que dans la protolangue il y avait des phrases coordonnées sans conjonction, comme nous en trouvons des exemples dans les langues finno-ougriennes actuelles. Dans chacune de ces langues on trouve également des exemples pour la subordination sans conjonction. Nous pouvons en conclure que la protolangue distingue la coordination et la subordination seulement selon le contenu ; quant à la forme, seule la juxtaposition sans conjonction était connue.

\footnotetext{
${ }^{1} \mathrm{Si} \mathrm{nous} \mathrm{ne} \mathrm{les} \mathrm{citons} \mathrm{pas} \mathrm{dans} \mathrm{le} \mathrm{présent} \mathrm{article,} \mathrm{c'est} \mathrm{à} \mathrm{cause} \mathrm{des} \mathrm{difficultés} \mathrm{qui} \mathrm{se} \mathrm{posent} \mathrm{dans} \mathrm{la}$ transcription de sons reconstruits et disparus.
} 
D'après ces données on fait l'hypothèse (Haader 1991) que la subordination existait déjà en tant que rapport, mais sans conjonction. Si en proto-hongrois il n'existe probablement pas de conjonction, on note que dans les langues samoyèdes il n'en existe même pas jusqu'à nos jours. On suppose la présence de quelques relatifs déjà dans la période ougrienne, mais parmi les conjonctions que l'on retrouve actuellement en vogoul et en ostyak certaines ont apparu sous l'influence du russe, donc ce n'est pas un héritage finno-ougrien. Faute de documents écrits (Bárczi 1975, 175) il est difficile de savoir quand une conjonction est née, mais ce qui est sûr c'est que la traduction des périphrases dans des textes latins du $16^{\mathrm{e}}$ siècle a favorisé la multiplication des conjonctions.

Comme nous n'avons pas de conjonctions communes avec les langues apparentées ${ }^{2}$, nous sommes amenés à considérer la période du proto-hongrois comme l'époque de l'apparition de ces outils grammaticaux (Haader 2003).

Les changements les plus importants qui se déroulent à cette période sont les suivants :

- apparition des types de subordonnées les plus importants,

- apparition de certaines conjonctions,

- apparition de certains mots de renvoi (ou : «antécédents », terme figurant dans les grammaires du hongrois destinées aux francophones). La conséquence de l'apparition de ces éléments est que le rapport entre les propositions devient plus étroit.

Étant donné qu'il s'agit d'un phénomène inconnu en français, il semble nécessaire d'ouvrir une parenthèse sur l'importance des "antécédents » ${ }^{3}$. Si le phénomène semble inconnu, il est d'autant plus difficile de se fixer sur la terminologie. Les grammaires du hongrois rédigées en français utilisent le terme antécédent, mais on pourrait également les appeler mots de référence ou mot de renvoi. Il s'agit (Nyéki 1998, 228) de la présence quasi-obligatoire dans la principale d'un antécédent pronominal ou adverbial, qui sert de point d'appui pour la subordonnée. Comme on le voit dans les exemples dans (1), il s'agit d'un démonstratif décliné.

1a) Megueszem ąt

a könyvet,
DEM-ACC le livre
n' j'ai parlé.]

\section{amelyikeröl} beszéltem

J'achète

RELin-DELAT

parler-PA-1SG

['achète le livre dont j'ai parlé.]

1b) Aqt mesélik, bogy Pétert

elóléptetik.

DEM-ACC raconter-3PL que Pierre-ACC promouvoir-3PL

[On raconte que Pierre sera promu]

1c) Azért iszom, mert szomjas vagyok.

DEM-FIN boire-1SG, parce que assoiffé être.1SG

[Je bois parce que j'ai soif.]

\footnotetext{
${ }^{2}$ Seules les bases pronominales du type ho-/ ha sont communes.

3 Les énoncés présentés sont partiellement empruntés à Szende et Kassai (2001), avec quelques modifications ou simplifications.
} 
1d) Azért bivtak meg, bogy elóadást tartsak. DEM-FIN inviter-PA- 3PL PREV que exposé-ACC faire-SUBJ.1SG ['ai été invité pour que je fasse un exposé]

Après les généralités, nous nous approchons des subordonnées à partir de deux points qui semblent être très importants en hongrois :

1) les constructions participiales ayant rempli dans un premier temps la fonction qui sera plus tard celle des subordonnées conjonctives et

2) les relatives qui ont été les premières subordonnées à se former et à propos desquelles on arrive à l'analyse des conjonctions dont la plupart ont leur origine dans les pronoms relatifs.

\subsection{Les constructions participiales}

Pendant les époques précoces de l'histoire du hongrois et des langues apparentées, la fréquence des constructions participiales était prédominante (Haader 1991, 2003). L'expression du contenu subordonné était réalisée par des formes nominales du verbe (infinitifs ou participes accompagnés de compléments liés à un verbe conjugué). Ces constructions ont progressivement disparu, définitivement vers le $16^{\mathrm{e}}$ siècle.

\subsection{Les relatives}

Les premières subordonnées à apparaître sont probablement les relatives dans lesquelles l'élément relatif est un interrogatif-indéfini. L'hypothèse généralement acceptée dans les grammaires historiques du hongrois est la suivante (voir Juhász 1991) :

2) Ki éhes? - Egyék. $\rightarrow$ aki éhes egyék (Ha) valaki éhes egyéke.

Qui avoir-faim ? - Qu'il mange $\rightarrow$ (Celui) qui a faim, qu'il mange $\rightarrow$ (si) qqn a faim, qu'il mange

$K i$ pour l'humain et mi pour inanimé sont d'abord des interrogatifs. Les différentes couches du sens sont donc : interrogatif - relatif - indéfini. Selon Juhász (op. cit.) la mise en rapport des deux propositions simples se fait certainement à l'aide de l'intonation.

\section{L'origine des subordonnants}

Rappelons que dans la connaissance des langues apparentées qui n’utilisent pas de conjonctions, on peut affirmer que les conjonctions de subordination représentent un développement propre à la langue hongroise. 


\subsection{La morphologie des conjonctions}

En hongrois (Juhász 1991) on distingue des formes simples, des formes composées et des conjonctions formées de plusieurs lexèmes que nous appellerons conjonctions complexes. En fait, cette distinction n'est pas suffisamment transparente dans la mesure où par formes composées on entend en diachronie celles qui se composent d'une base pronominale et d'un suffixe casuel. Les formes simples sont le plus souvent des conjonctions de coordination (es 'aussi', de 'mais'), mais il y en a parmi les subordonnants également ( $h a$ 'si').

\subsection{Les catégories source}

Selon les études translinguistiques (Kortmann 1997, 2001) et celles qui ont été réalisées sur le français (entre autres Bat Zeev et Kemmer 1988), on peut observer des tendances générales qui sont valables également pour le hongrois, notamment le fait que les conjonctions se forment à partir de catégories lexicales existantes, par un changement qui peut être considérée comme un processus de grammaticalisation. Les catégories source, extrêmement homogènes, sont le plus souvent les suivantes :

- adverbes

- relatifs et interrogatifs

- adpositions (pré- ou postpositions)

- noms (simples ou déclinés)

- verbes.

Toutefois, et c'est ce qui est important, comme les adverbes et les adpositions sont des catégories mineures, il faut remonter plus loin dans leur histoire. Concernant le hongrois, cela nous amène jusqu'aux bases pronominales et aux relatives. En effet, selon Bárczi et al. $(1967,393)$, la plupart des conjonctions de subordination ont une origine de pronom relatif : ki 'qui', mi et mely, 'quoi', ou d'adverbe relatif : hol 'où', mikor 'quand"4 et on les appelle dans la terminologie grammaticale du hongrois " conjonctions relatives ». C'est un terme qui n'est pas utilisé dans les grammaires du français, mais il semble utile dans la description du hongrois. Par conséquent, on pourrait parler en général des «subordonnants ». Nous en présenterons quelques-uns, en particulier ceux dont nous pouvons bien suivre l'évolution. Les plus anciens sont les relatifs d'origine interrogative, $k i$ 'qui' et $m i$ 'qu'est-ce qui', ensuite hogy 'que', puis ha 'si'.

\subsubsection{Les éléments qui remontent à la base pronominale mi-}

Les pronoms relatifs (Bárczi et al. 1967, 210) que nous utilisons comme conjonctions de subordination sont probablement les plus jeunes parmi les pronoms. Les pronoms relatifs les plus précoces ont non seulement les mêmes formes que les interrogatifs-indéfinis mais ils en sont issus. On ne sait pas encore quand le pronom

4 Dans la langue moderne, ces éléments peuvent commencer par un a-, qui est à l'origine un démonstratif et qui s'y est joint vers le 16 ème siècle. 
démonstratif az (devant consonne a) commence à s'y antéposer, probablement vers le $15^{\text {ème }}$ ou $16^{\text {ème }}$ siècle. Il s'agit donc des subordonnants tels que :

3) (a)ki

(a) $m$

$\operatorname{mint}$

'qui [+hum]'

mig

'qui $[-$ an]'

'comme'

mikor (< mikoron $\left.15^{\mathrm{e}} \mathrm{mi}+\mathrm{kor}+\mathrm{on}\right)$ 'quand, puisque'

mibelyt

'pendant que, tandis que'(m- +ig 'jusqu'à')

mivel $($ mi+vel $=$ comitatif)

'aussitôt que'

mert

'puisque'

'parce que', etc.

Voici quelques remarques sur ces éléments :

- (a)mikor 'quand' : cet exemple représente la grammaticalisation en termes de sens TEMPOREL $>$ CAUSE et CONDITION.

- mert 'parce que' est un élément composé d'un relatif mi- et d'un suffixe causal-final:

mi + ért $>$ miért $>$ mért $>$ mërt $>$ mert.

Un exemple reconstruit forgé peut expliquer l'évolution :

4a) amiért bántottad azért baragszile ce pourquoi/tu lui as fait mal/c'est la raison pour laquelle/il est fâché

L'ordre original des propositions a pu être subordonnée - principale et il a changé en principale - subordonnée, ce qui correspond au changement de l'ordre cause - effet en effet - cause :

4b) Haragszik, mert bántottad

Il est fâché/parce que (car)/tu lui as fait mal

Ainsi, le rapport entre les deux propositions (Juhász 1991) et la conjonction même évoluent vers la coordination. En effet, actuellement, mert est classé avec les conjonctions de coordination, puisqu'il s'agit plutôt de donner une explication, non une cause. C'est à peu près la même opposition que l'on propose en français entre car et parce que (ou entre denn et weil en allemand où la situation se complique concernant l'ordre des mots). Il semble qu'en hongrois le seul critère formel qui permet de distinguer entre coordination et subordination reste celui qui teste la possibilité de mettre en tête de phrase l'élément en question : seule une conjonction de subordination peut commencer la phrase complexe, la conjonction de coordination reste entre les deux phrases reliées.

L'élément

- $\quad$ mint 'comme, comment' 
est une conjonction composée, d'une base de pronom relatif et d'un affixe modal: $m i+-n t$, le sens est mode et état. Dans des textes précoces c'est encore le sens relatif qui domine, l'élément apparaît plus tard avec des verbes ismer 'connaître' et tud 'savoir' :

5) mynt evt esmeriuc

mint őt ismerjük

comme 3SG-ACC connaître-1PL

[comme nous le connaissons]

\subsubsection{Les éléments qui remontent à la base pronominale ho-}

Il s'agit d'une base (Bárczi et al. 1967, 209) d'origine finno-ougrienne (bo-, ba-) qui n'a pas survécu au nominatif; mais dans d'autres cas ils sont représentés par des suffixes adverbiaux très anciens, notamment locatifs, d'où les formes :

bol 'où localisation', hová 'où direction', honnan 'd'où', etc.

Ce sont des adverbes relatifs spatiaux, mais il faut noter ici une particularité de la tradition grammaticale du hongrois, dans la mesure où on distingue des «subordonnées circonstancielles locatives » ce qui n'est pas d'usage pour le français. D'autres conjonctions remontent également à cette base, notamment ha 'si' et hogy 'que'.

L'élément le plus proche du français que est la conjonction hogy qui est pourtant utilisé plus largement : il peut être en co-occurrence avec un élément interrogatif dans l'interrogation indirecte. Quant à son origine, deux hypothèses sont proposées (Haader 91, 729), d'une part une origine interrogative :

6) Hallom, [hogyan $\rightarrow$ hogy] könyörögsz.

[j'entends comment tu supplies]

Une autre hypothèse propose à l'origine un adverbe qui avait pu être utilisé comme relatif :

7) Hallom [ahogyan $\rightarrow$ hogy] könyörögsz. [j'entends la-manière-dont tu supplies]

Par la suite de l'évolution, le sens de cet élément s'est affaibli et il est devenu une conjonction générale, vide. A l'origine de cette conjonction on retrouve effectivement un cas de changement de catégorie, à savoir celui où un adverbe devient conjonction.

\section{D'autres types d'évolution}

Un autre ensemble remonte à une forme verbale: la conjonction biszen 'comme, puisque' remonte à une construction verbale : 
8) hiszem (ąt, [hogy]) > hiszen

je crois ceci que $>$ puisque

Toutefois, si l'on soumet cette conjonction au test de la position en tête, il semble qu'il s'agit plutôt d'une conjonction de coordination qui n'accepte pas cette position initiale.

La conjonction de concession et d'opposition la plus fréquente même de nos jours remonte probablement (Juhász 1991) à un adverbe non affixé qui signifie 'courageusement' (de bátor 'courageux'), avec une reconstruction de sens : on peut permettre, courageusement, l'affirmation qui suit, quoique son sens aille à l'encontre de nos présuppositions. L'évolution probable ${ }^{5}$ de la forme est la suivante :

9) bár 'quoique, bien que' < ámbár < ámbátor < bátor

\section{Les changements sémantiques}

L'observation du développement de la morphologie de ces éléments ne nous permet pas encore de suivre le développement sémantique. En effet, selon Bárczi et al. (1967, 468), certaines conjonctions d'origine relative ont développé un contenu sémantique spécifique qu'une relative ne pouvait pas exprimer, à savoir

- $\quad$ comparative : mint 'comme'

- consécutive : hogy 'que'

- conditionnelle : $h a$ 'si', etc.

Il convient donc d'étudier à part le développement sémantique. Il semble que certaines tendances observées par ceux qui travaillent sur les processus de grammaticalisation peuvent être illustrées au cours de l'évolution du hongrois également.

\subsection{Les développements ESPACE > TEMPS et TEMPS > CAUSE}

Le développement ESPACE >TEMPS peut être illustré dans plusieurs catégories lexicales ${ }^{6}$, notamment parmi les postpositions ou les adverbes, mais également parmi les conjonctions qui nous intéressent :

$$
\begin{aligned}
& \text { 10a) mielótt } \\
& \text { mi+előtt } \\
& \text { 'quoi+devant' } \\
& \text { 'avant que' }
\end{aligned}
$$

${ }^{5}$ Pour tous les linguistes cette explication ne paraît pas très convaincante (pers. comm. de B. Boiron).

${ }^{6}$ Voir Sörés 2006, chap. VI. 
10b) miután

mi+után

'quoi+après »

'après que'

10c) mihelyt

$\mathrm{mi}+$ hely $+\mathrm{t}$

quoi+lieu+locatif

'aussitôt que' savoir :

De même, d'autres évolutions peuvent paraitre «typologiquement banales », à

TEMPS $>$ CAUSE, représenté par mikor 'quand' et

ESPACE $>$ TEMPS $>$ CAUSE, miután 'puisque'.

La chaîne ESPACE $>$ TEMPS peut aboutir également à la CONCESSION, dans holott 'quoique', etc.

\section{Influence du latin, aspects culturels}

Dans ce qui précède, nous avons vu un certain nombre de conjonctions de subordination qui sont utilisées encore en hongrois moderne et dont on peut suivre l'évolution. Toutefois, on observe au cours de l'évolution que le $17^{\text {ème }}$ siècle a connu un développement important, voire une prolifération de conjonctions ayant la même fonction. Il y en a de nouvelles qui ont survécu mais il y en a beaucoup qui ont disparu, en particulier celles qui ont une forme complexe, celles qui se composent de plus d'un lexème. Ce phénomène illustre évidemment le «style baroque » de l'époque, comme on le retrouve au cours de l'histoire d'autres langues dont le français. Étant donné que pour le hongrois il s'agit d'une catégorie lexicale qui n'a pas été héritée mais développée au cours de l'histoire de la langue, on peut se poser quelques questions sur cette évolution. Le hongrois, malgré ses origines géographiquement éloignées, s’inscrit dans l'espace culturel de l'Europe et en particulier dans le rayonnement culturel du latin. Il ne s'agit pas d'emprunt directs au latin, mais de l'influence qu'ont pu subir certaines constructions et certains éléments, dont les conjonctions. Comme nous l'avons mentionné au début, la plupart des textes précoces, et la plupart des manuscrits à partir du $15^{\text {ème }}$ siècle sont des traductions du latin où certaines interférences semblent inévitables. D'une part on a vu que dans la langue ancienne les constructions participiales étaient beaucoup plus fréquentes que de nos jours. Il a également été observé, surtout au $16^{\text {ème }}$ siècle, que les constructions accusativus cum infinitivo sont traduites fidèlement. Ces constructions ont finalement disparu et la fréquence des subordonnées participiales a également diminué. On peut donc supposer que ce n'est pas directement l'influence du latin qui a agi sur le développement et, à certaines époques, a suscité la prolifération des conjonctions de subordination, mais qu'il s'agirait plutôt d'une tendance générale de l'écrit. 


\section{La place du hongrois dans l'étude typologique de Kortmann (1997)}

\subsection{Le prototype de la conjonction adverbiale. Les sources. Les formes}

L'étude de Kortmann $(1997,72)$ propose un prototype qui lui sert de base pour sa comparaison. Ainsi, il s'agit

1. d'une forme libre non fléchie

2. qui opère sur une subordonnée finie ;

3. elle n'a pas de fonction syntaxique ;

4. a une position fixe à l'une des extrémités de la phrase sur laquelle elle opère.

Les propriétés 1 et 3 permettent de distinguer les conjonctions «adverbiales » des pronoms relatifs, ce qui est important car au niveau de la syntaxe on observe que, à cause d'une homonymie, la distinction d'une relative et d'une circonstancielle pose parfois des problèmes (cf. Creissels 2006, II : 192-193). Notons que la propriété 3 portant sur la fonction devrait être précisée si l'on considère comme fonction celle que remplit un adverbe ou une circonstancielle en tant que modifieur adverbial optionnel dans la phrase. En plus, ce prototype ne permet pas de faire la distinction entre circonstancielle et complétive, voir l'objection de Creissels (id.) sur une causale introduite par pour que qui pourrait également être considérée comme un complétive complément de préposition.

En considérant dans leur ensemble les langues étudiées par Kortmann, donc y compris les deux langues classiques éteintes, les sources les plus importantes des conjonctions sont les suivantes : interrogatifs, relativiseurs, adverbes, surtout en latin et en grec ancien, ce à quoi s'ajoutent dans les descendants modernes les prépositions et les complémenteurs.

Selon ses observations, dans les 49 langues étudiées, 95 pour cent des conjonctions adverbiales peuvent prendre les formes suivantes :

- un morphème monosyllabique

- un morphème polysyllabique

- plus d'un morphème dans un mot

- plus d'un mot.

Il se pose la question de savoir si les formes représentées par le hongrois peuvent s'intégrer à ce schéma.

\subsection{Les propriétés $d u$ hongrois}

\subsubsection{Morphologie}

Avant de réaliser son analyse, Kortmann (1997) pose l'hypothèse de l'existence d'une région centre et d'une région périphérique dans l'aire géographique étudiée.

\footnotetext{
${ }^{7}$ La cinquième place est celle des conjonctions discontinues dont on ne tiendra pas compte dans la présente étude. En effet, il semblerait, à partir d'un bref parcours du hongrois, que ce sont surtout les conjonctions de coordination qui se présentent sous forme discontinue.
} 
Cette distinction ne s'avère pas opérationnelle, puisqu'il n'y a pas une des langues de périphérie qui ne partagerait au moins un trait avec les langues du centre.

Un deuxième point de comparaison des langues consiste à étudier les corrélations entre certaines caractéristiques typologiques et les propriétés qu'elles présentent concernant les conjonctions. Deux traits syntaxiques ont été choisis : l'ordre des mots, en l'occurrence les trois ordres les plus représentés parmi les langues du monde, à savoir SVO, SOV et VSO, ainsi que la présence de prépositions ou de postpositions. Le hongrois est ainsi considéré comme une langue SOV, postpositionnelle.

Dans mon ouvrage intitulé Le hongrois dans la typologie des langues (2006) j’ai réussi à démontrer qu'au niveau des constituants de la phrase le hongrois est une langue SVO, c'est l'ordre non marqué. Toutefois, l'ordre des mots dans les syntagmes et la plupart des autres traits sériels permettent effectivement de classer le hongrois parmi les langues du type OV (déterminant - déterminé avec la terminologie française), ce qui est en corrélation avec la présence de postpositions.

Selon les tableaux récapitulatifs, malgré le fait qu'il est le seul à être du type OV au centre, le hongrois semble appartenir au centre supposé, selon le fonctionnement des conjonctions. Toutefois, pour mieux comprendre ce fonctionnement, il faut tenir compte d'un autre trait typologique qui n'est pas mentionné dans cette étude translinguistique, à savoir la morphologie majoritairement agglutinante du hongrois. Ce fait explique un certain nombre de phénomènes, ou au moins il les met sous une lumière différente.

Du côté morphologique, on observe que la première sous-classe des conjonctions, les monosyllabiques, à savoir ha, hogy, mert, mint, sont synchroniquement indécomposables. Mais diachroniquement une segmentation est possible, comme on l'a vu dans la première partie de notre article. Le résultat des segmentations modifie l'une des constatations de Kortmann; en effet, selon la carte 8.11. le hongrois se distingue des autres langues du centre en n'ayant qu'une faible proportion de conjonctions qui remontent à un relatif/relativiseur.

En ce qui concerne les conjonctions polysyllabiques, noha, mivel, holott elles peuvent se décomposer en diachronie, mais il faut souvent remonter à des périodes très anciennes de la langue.

Les conjonctions polymorphématiques (mialatt, miköz̧ben, miután, mihelyt, mintha, mintsem, amennyiben, amennyire) sont plus facilement segmentables, étant donné le caractère agglutinant de cette langue :

- Segmentables en REL-Postp :

mi+alatt, mi+köz̧ben, mi+szerint, mi+után, mi+elött, mi+által

- Segmentables en DEM-Postp :

a+nélkül, a+belyett

Pour ce qui est des conjonctions qui se composent de plusieurs mots et qui sont souvent accompagnées, comme dans beaucoup de langues, d'un complémenteur, notamment de hogy, elles sont de formation ultérieure, moderne, parfois condamnées comme trop compliquées, parfois considérées comme des germanismes. 
Complémenteur incorporé :

alighogy, ahogy, minthogy, mivelhogy, nehogy, hogyha

Complémenteur séparé/ plusieurs mots :

azóta, hogy ; feltéve, hogy ; annak ellenére, hogy : azért, hogy ; azóta, hogy ; úgy, hogy.

\subsubsection{Polyfonctionalité}

Dans l'étude typologique mentionnée, il est question du caractère éventuellement polyfonctionnel des conjonctions. Il peut s'agir de polyfonctionalité syntaxique et de polysémie. Au sujet du hongrois, ces deux facteurs ne paraissent pas être d'une grande importance. En effet, il n'y a pas de polyfonctionalité syntaxique entre les conjonctions et les postpositions puisque ces dernières sont incorporées dans les premières. Seuls quelques éléments peuvent fonctionner avec la même forme comme adverbes et conjonctions à la fois, amikor 'quand', mikor 'quand' interrogatif, mivel 'avec quoi ?', etc.

En revanche, la polysémie est beaucoup plus riche, tout en ne manifestant pas de particularités très intéressantes du point de vue typologique. En effet, il s'agit des conjonctions qui ont connu des changements sémantiques du type ESPACE > TEMPS $>$ AUTRE CONCEPT et quelques-unes ont maintenu, à part le sens spatial, les autres sens acquis :

hogy : conséquence, cause, but, manière

miután: temps, cause

(a)mikor: temps, cause, contraste

mig : temps, contraste

\subsubsection{Disparition et renouvellement}

Certaines époques ont vu la multiplication de conjonctions polymorphématiques voir polylexématiques, notamment celles qui se composent de trois éléments, mais elles n'ont pas connu une longue vie dans l'histoire de la langue.

Les observations qui portent sur les tendances du hongrois moderne signalent le processus au cours duquel apparaissent de nouvelles postpositions, mais il n'y a aucune mention d'éventuels changements au sujet des conjonctions. Quant aux registres, un certain nombre d'éléments sont jugés littéraires ou soutenus, comme miáltal, minélfogva, minthogy, ámbár, etc., d'autres littéraires ou clairement désuets, comme midôn ou bogysem. 


\section{Tableau récapitulatif}

Le tableau suivant permet de voir l'ensemble des conjonctions de subordination du hongrois dont il était question plus haut. Certaines ne sont utilisées que dans un registre soutenu, en particulier les concessives.

\begin{tabular}{|c|c|c|c|}
\hline \multirow[t]{14}{*}{ Temps } & Simultanéité & (a)mikor & quand, lorsque \\
\hline & & ameddig, amíg & jusqu’à ce que \\
\hline & & Míg & tant que, pendant que \\
\hline & & mialatt & pendant que \\
\hline & & miközben & pendant que \\
\hline & & mígnem & jusqu'à ce que \\
\hline & Antériorité & miután & après que \\
\hline & & amint & dès que, aussitôt que \\
\hline & & mihelyt & aussitôt que \\
\hline & & ahogy & aussitôt que \\
\hline & & alighogy & à peine que \\
\hline & Postériorité & mielőtt & avant que \\
\hline & & (a)mióta & depuis que \\
\hline & & azóta, hogy & depuis que \\
\hline \multirow[t]{2}{*}{ Cause } & Cause & mert & parce que \\
\hline & & minthogy (soutenu) & $\begin{array}{l}\text { parce que, puisque, vu que, } \\
\text { attendu que }\end{array}$ \\
\hline \multirow{10}{*}{ Concession } & & hogy & Que \\
\hline & & mivel & Puisque \\
\hline & & mivelhogy (arch.) & Puisque \\
\hline & & mikor & puisque, quand \\
\hline & & miután & puisque, vu que \\
\hline & Condition & $\mathrm{Ha}$ & $\mathrm{Si}$ \\
\hline & & feltéve, hogy & supposé que \\
\hline & & hogyha & $\mathrm{Si}$ \\
\hline & & amennyiben & si, dans la mesure où \\
\hline & Concession & Bár & bien que, quoique \\
\hline
\end{tabular}




\begin{tabular}{|c|c|c|c|}
\hline & & ámbár (soutenu) & bien que, quoique \\
\hline & & noha (soutenu) & bien que, quoique \\
\hline & & jóllehet (soutenu) & bien que, quoique \\
\hline & & $\begin{array}{l}\text { holott (pas en tête de } \\
\text { p) }\end{array}$ & bien que, quoique, pourtant \\
\hline & & annak ellenére, hogy & malgré (le fait que) \\
\hline & Contraste & Míg & alors que, tandis que \\
\hline & But & hogy & pour que \\
\hline & & azért, hogy & pour que, afin que \\
\hline & & nehogy & pour ne pas que, de peur que \\
\hline & Conséquence & hogy & Que \\
\hline & & ugyhogy & de manière que \\
\hline & Comparaison & $\operatorname{mint}$ & Comme \\
\hline & & mintsem & que ... ne (plutôt que ?) \\
\hline \multirow[t]{4}{*}{ Modal } & Manière & anélkül, hogy & sans que \\
\hline & & (a)hogy & de la manière que, comme \\
\hline & & amennyire & pour autant que \\
\hline & & hogysem & plutôt que \\
\hline \multirow[t]{3}{*}{ Autres } & Instrum. & miáltal (soutenu) & ce par quoi \\
\hline & Place & Hol & Où \\
\hline & Substitution & ahelyett, hogy & au lieu \\
\hline
\end{tabular}

\section{Conclusions}

L'objectif de cet article a été de présenter des faits observables en hongrois et de trouver la place de cette langue dans une typologie. On a observé que, contrairement à ce qu'affirment les approches translinguistiques, on ne peut pas chercher l'origine de la plupart des conjonctions de subordination directement dans un adverbe, puisque les éléments source ont le plus souvent une origine pronominale, en particulier une source de pronom relatif ou démonstratif. Ainsi, le processus de grammaticalisation qui se déroule n'est pas du type lexème > grammème, mais plutôt élément moins grammatical > élément plus grammatical.

Du point de vue morphologique, il parait que l'on n'a pas intérêt à distinguer entre conjonctions polysyllabiques et polymorphématiques. En effet, certaines segmentations ne sont pas explicables en synchronie, malgré le caractère agglutinant du hongrois. Mais lorsqu'il est possible de segmenter les conjonctions déjà formées, il est plus facile de raisonner sur leur sens également, par exemple sur la participation du démonstratif au sens, que l'on retrouve également en français, sous la forme de ce mais, 
vu la différence des techniques morphologiques, en français cela se présente sous forme d'un élément autonome.

Le travail réalisé présente en même temps des perspectives: une analyse plus approfondie des conjonctions de subordination dans une approche typologique est prévue.

Abréviations :

ACC $=$ ACCUSATIF, DEM $=$ DEMONSTRATIF, DELAT $=$ DELATIF, FIN $=$ CAUSAL-FINAL, $\mathrm{PA}=$ PASSE, $\mathrm{PL}=$ PLURIEL, POSTP $=$ POSTPOSITION, PREV $=$ PREVERBE, $\mathrm{REL}=(\mathrm{PRONOM})$ RELATIF, RELin=PRONOM RELATIF INANIME, $\mathrm{SG}=$ SINGULIER, SUBJ $=$ SUBJONCTIF/CONJONCTIF.

\section{RÉFÉRENCES BIBLIOGRAPHIQUES}

BARCZI G., 1975², A magyar nyelv életrajza, [Bibliographie de la langue hongroise], Budapest, Gondolat Kiadó.

BARCZI G., 1991, Szófejtő szótár, [Dictionnaire étymologique], Budapest, Trezor Kiadó.

BARCZI G., BENKO L.\& BerRAR J., 1967, A magyar nyelv története [Histoire de la langue hongroise], Budapest, Nemzeti Tankönyvkiadó.

BAT-ZEEV SHYLDKROT H. \& KEMMER, S., 1988, «Le développement sémantique des conjonctions en français : quelques concepts généraux », Revue Romane, 23 : 1, p.920.

BENKO L., 1991, A magyar nyelv történeti nyelvtana [Grammaire historique de la langue hongroise], Budapest, Akadémiai Kiadó, vol. I.

BERECZKI G., 1996, A magyar nyelv finnugor alapjai [Les bases finno-ougriennes de la langue hongroise], Budapest, Universitas Könyvkiadó.

CREISSELS D., 2006, Syntaxe générale. Une introduction typologique, vol. 2., Paris, Lavoisier.

HAADER L., 1991, « Az alárendelő mondatok » [Les subordonnées], in Benkő Loránd, A magyar nyelv történeti nyelvtana [Grammaire historique de la langue hongroise], Budapest, Akadémiai Kiadó, vol. I, p.729-741.

HAADER L., 2003, « Az összetett mondat » [La phrase complexe], in Kiss Jenő et Pusztai Ferenc, Magyar Nyelvtörténet [Histoire de la langue hongroise], Budapest, Osiris Kiadó, p. 260-267.

HAGEGE Cl., 2001, "Les processus de grammaticalisation », in Haspelmath et al., Language Typology and Language Universals, Berlin-New York, De Gruyter, vol. II., p. 1609-1623.

JuHAsz D., 1991, A kötösrók [Les conjonctions], in Benkő Loránd, A magyar nyelv történeti nyelvtana [Grammaire historique de la langue hongroise], Budapest, Akadémiai Kiadó, vol. I, p.476-500. 
Anna Sörés

KISS J. \& PUSZTAi F., 2003, Magyar nyelvtörténet [Histoire de la langue hongroise], Budapest, Osiris Kiadó.

Kortmann B., 1997, Adverbial Subordination, Berlin -New York, Mouton de Gruyter.

KorTmann B., 2001, "Adverbial conjunctions », in Haspelmath et al., Language Typology and Language Universals, Berlin-New York, De Gruyter, vol. I., 2001, p. 842854.

NYEKI L., Grammaire pratique du hongrois d'aujourd'bui, Paris, OPHRYS-POF, 1988,

SORES A.2006, Le hongrois dans la typologie des langues, Limoges, Lambert-Lucas.

SZENDE T .\& KASSAI G., 2001, Grammaire fondamentale du hongrois, Paris, l'Asiathèque. 